for myelofibrosis. N Engl J Med 2012 ; 366 (9) : 799-807.

[3] Pardanani A, Harrison C, Cortes JE, et al. Safety and efficacy of fédratinib in patients with primary cal trial. JAMA Oncol 2015 ; 1 (5) : 643-51.

[4] Mesa RA, Vannucchi AM, Mead A, et al. Pacritinib versus best available therapy for the treat- ment of myelofibrosis irrespective of baseline cytopenias (PERSIST-1): an international, randomised, phase 3 trial. Lancet Haematol 2017 ; 4 (5) e225-36

\title{
Adjonction du daratumumab à l'association bortézomib, lénalidomide et dexaméthasone dans le myélome multiple éligible à une autogreffe de cellules souches hématopoiétiques
}

\section{Nicolas Stocker}

'association du bortézomib, du lénalidomide et de la dexaméthasone (VRD) est un standard de traitement de première ligne des patients atteints de myélome multiple nouvellement diagnostiqués (MMND) et éligibles à une autogreffe de cellules souches hématopoïétiques, permettant d'obtenir une médiane de survie sans progression de 50 mois et un taux de survie globale de $81 \%$ à quatre ans [1]. Récemment, l'étude CASSIOPEIA a démontré que l'adjonction du daratumumab (Dara), un anticorps monoclonal anti-CD38, au traitement d'induction associant $\mathrm{du}$ bortézomib, de la thalidomide et de la dexaméthasone améliorait significativement son efficacité chez ces patients [2]. Les auteurs de cette étude de phase II ont donc évalué l'adjonction du Dara au VRD dans ce contexte clinique [3].

Entre décembre 2016 et avril 2018, 207 patients ont été inclus dans cette étude multicentrique de phase II ayant comparé le VRD au Dara-VRD (104 patients dans le groupe DaraVRD, 103 patients dans le groupe VRD). Le critère de jugement principal était le taux de réponse complète (RC) stringente au terme de la consolidation. Les patients recevaient quatre cycles d'induction de 21 jours, une intensification thérapeutique par melphalan $\left(200 \mathrm{mg} / \mathrm{m}^{2}\right)$ suivie d'une autogreffe de cellules souches hématopoïétiques, puis deux cycles de consolidation de 21 jours chacun. Les groupes de traitement étaient équilibrés avec une médiane d'âge de 60 ans (29-70) et un délai médian depuis le diagnostic de 0,8 mois (0-61). Près de la moitié des patients présentaient un stade ISS (pour international staging system) I (48\%) et $16 \%$ des patients $(\mathrm{n}=30)$ présentaient un myélome multiple de haut risque cytogénétique (délétion du $17 \mathrm{p}$, translocations $[4,14]$ ou $[14,16])$.

Au terme des cycles de consolidation, le taux de RC dans le groupe Dara-VRD était supérieur à celui du groupe VRD (42 versus $32 \%$, OR : 1,57 ; 95\%CI : $0,87-2,82 ; \mathrm{P}=0,068$ avec un risque $\alpha$ de 0,10$)$. Les taux de réponse globale et de réponse de très bonne qualité à ce stade étaient significativement supérieurs dans le groupe Dara-VRD (99 versus $92 \% ; \mathrm{P}=0,016$ et 91 versus $73 \% ; \mathrm{P}=0,001)$. Avec un suivi médian de 22 mois, ces résultats étaient conservés, tant en termes de taux de RC (65 versus $45 \%$, OR : 1,98; 95\%CI : 1,12-3,49; $\mathrm{P}=0,01$ ) qu'en termes de taux de RC stringente (80 versus $61 \%$, OR 2,53 ; 95\%CI : 1,33$4,81 ; \mathrm{P}=0,005)$. Les taux de maladie résiduelle (MRD) indétectable (MRD : $10^{-5}$ ) dans le groupe Dara-VRD étaient significativement supérieurs à ceux du groupe VRD (respectivement 51 versus $20 \%$, P $<0,0001)$. En revanche, les taux de RC stringente et de MRD indétectable n'étaient pas différents dans les sous-groupes de patients présentant un score ISS à 3 et/ou un haut risque cytogénétique.

Sur le plan de la tolérance, les principaux effets indésirables de grade 3-4 dans les groupes DaraVRD et VRD étaient des neutropénies (41 versus $22 \%$ ), des lymphopénies (23 versus $22 \%$ ), des thrombopénies (16 versus $9 \%$ ), des leucopénies
(16 versus $9 \%$ ), et des pneumopathies (8 versus $11 \%$ ). L'incidence des infections de grade 3-4 était comparables entre les groupes (23 versus $22 \%$ ) et ces dernières engendraient une interruption de traitement chez respectivement 2 et $3 \%$ des patients. Quarante-deux pour cent (42\%) des patients du groupe Dara-VRD présentaient une réaction à l'infusion du Dara (seulement $6 \%$ de réaction de grade III, aucune de grade 4-5). Ces réactions concernaient majoritairement la première administration du traitement.

En conclusion, l'association DaraVRD permet un gain significatif en profondeur de réponse lors du traitement de première ligne des patients atteints de MMND, éligibles à l'autogreffe de cellules souches hématopoiétiques (à l'exception des patients avec un score ISS à 3 et/ou un haut risque cytogénétique), avec un profil de toxicité acceptable. Des essais randomisés prospectifs permettront de mieux étudier cette association qui pourrait prochainement devenir le standard de première ligne dans ce contexte. ]

\section{Références}

[1] Attal M, Lauwers-Cances V, Hulin C, et al. Lenalidomide, Bortezomib, and Dexamethasone with Transplantation for Myeloma. N Engl J Med 2017 ; 376 : 1311-20.

[2] Moreau P, Attal M, Hulin C, et al. Bortezomib, thalidomide, and dexaméthasone with or without daratumumab before and after autologous stemcell transplantation for newly diagnosed multiple myeloma (CASSIOPEIA) : a randomised, open-label, phase 3 study. Lancet 2019 ; 394 : 29-38.

[3] Voorhees PM, Kaufman JL, Laubach J, et al. Daratumumab, lénalidomide, bortézomib, and dexaméthasone for transplant-eligible newly diagnosed multiple myeloma : the GRIFFIN trial. Blood $2020 ; 136: 936-45$ 